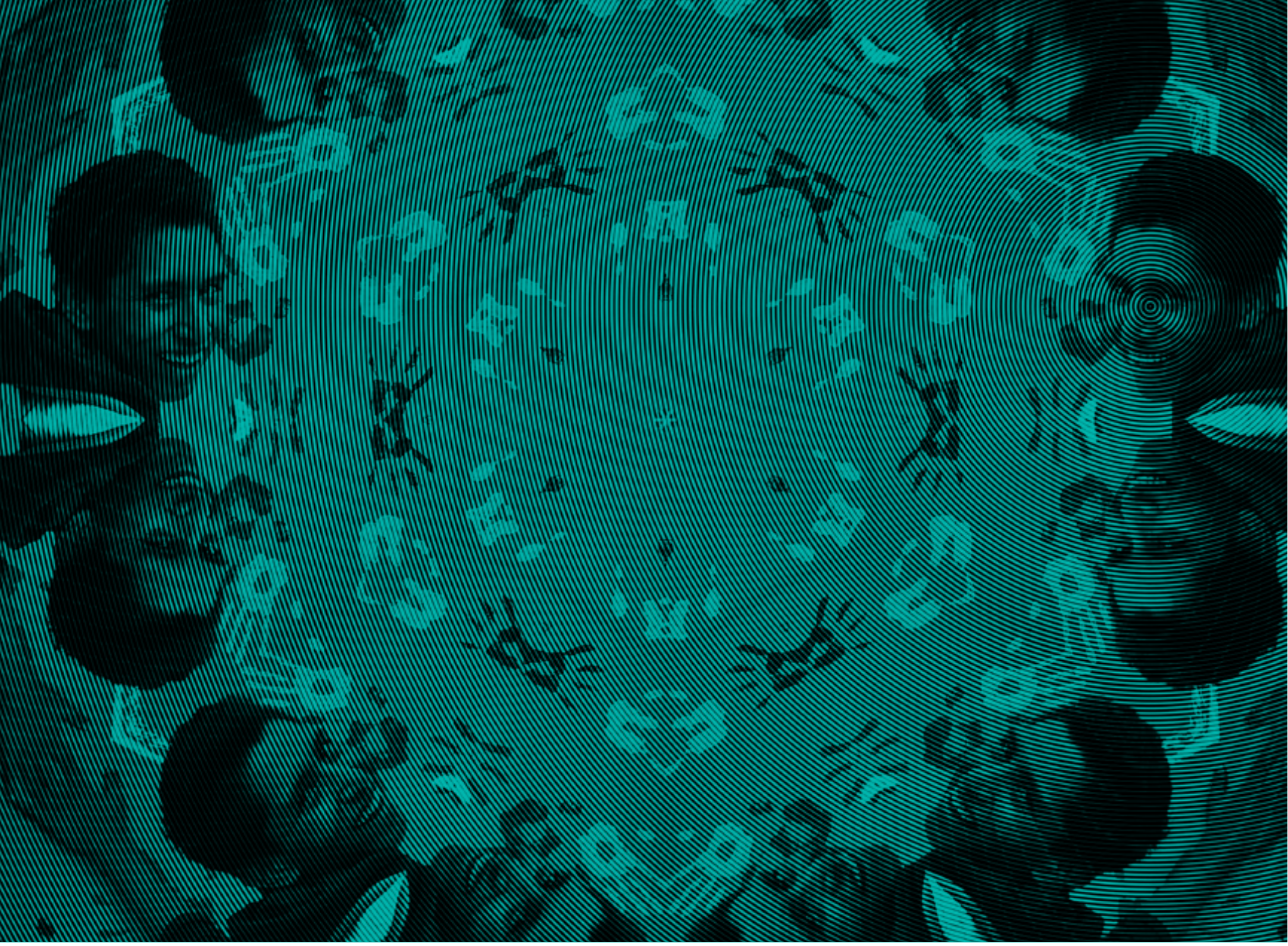

\title{
Atributos que humillan. Un enfoque relacional sobre las humillaciones entre estudiantes de educación secundaria
}

\author{
Attributes that humiliate. A relational approach on \\ humiliations among high school students
}

Atributos que humilha. Uma abordagem relacional sobre as relacionais das humilhações entre alunos de educação secundária 
Agustina Mutchinick 1
1. Investigadora del Instituto de Investigaciones en Ciencias de la Educación, Universidad de Buenos Aires, y de la Facultad de Humanidades y Ciencias Sociales de la Universidad Nacional de Misiones. Doctora, Universidad de Buenos Aires, Área de Ciencias de la Educación; correo electrónico: agustinamutchinick@gmail.com

Fecha de recepción: 18 de abril de 2016 / Fecha de aceptación: 16 de septiembre de 2016

\section{Resumen}

El presente artículo expone los resultados de una investigación cualitativa que exploró las relaciones de humillación entre estudiantes de secundaria, particularmente de los atributos que hacen evidentes tales modos de vincularse, entendiendo que la producción de dichas modalidades de sociabilidad se da en un entramado de configuraciones específicas. La investigación analizó desde un enfoque relacional dos grupos escolares de dos escuelas secundarias estatales, empleando para ello entrevistas en profundidad, individuales y grupales, a los alumnos de dichos grupos. Los resultados señalan que los atributos básicos para el desarrollo de las humillaciones varían en las condiciones estudiadas; antes que cualidades universales, similares en los distintos contextos, los términos humillantes se definen en función de las relaciones de fuerza entre los grupos interdependientes.

Palabras clave: Violencia, humillaciones, configuraciones educación secundaria argentina.

\section{Summary}

The paper presents results of a qualitative and exploratory study that analyse the relations of humiliation among high school students. In particular, reference is made to the attributes on which manifest these humiliations. Understanding that it is in the framework of specific configurations where the production of these forms of sociability is structured, the research analyzed, from a relational approach, two school groups of two public high schools. We carry out individual and group interviews to the students of those school groups. The results indicate that the attributes vary in the studied configurations. More than universal qualities, similar in different contexts, humiliating terms are defined depending of power relations established between the interdependent groups.

Keywords: Violence, humiliations, configurations argentine high school.

\section{Resumo}

O trabalho apresenta resultados de um estudo qualitativo de caráter exploratório sobre as relações de humilhação entre os alunos do ensino médio. Em particular, ele faz referência aos atributos em que estes modos de ligação é manifesto. Entendendo que é no quadro de configurações específicas em que a produção dessas formas de sociabilidade está estruturado, a pesquisa analisou a partir de uma abordagem relacional dois grupos escolares de dois escolas públicas secundárias. Foram realizadas entrevistas individuais e em grupo em profundidade aos alunos desses grupos escolares. Os resultados indicam que os atributos sobre os que se baseian as humilhação variam nas configurações estudadas. Mais que qualidades universais, semelhante em diferentes contextos, termos humilhantes são definidos em função de as relações de poder estabelecidas entre os grupos interdependentes.

Palavras chave: Violencia, humilhações, configurações educação secundária argentina. 


\section{Introducción}

El tema de las violencias en el ámbito escolar ha adquirido recientemente una gran relevancia en los medios de comunicación masiva. La espectacularización de ciertos episodios como el uso de armas, abusos sexuales o golpizas ha hecho de la escuela, especialmente de la escuela secundaria estatal, un escenario atravesado por la "violencia física” (Brener y Kaplan, 2006). Sin embargo, diversos trabajos dan cuenta de que son las violencias no físicas (insultos, burlas, apodos ofensivos, discriminaciones, humillaciones, etc.), las que determinan el llamado "clima de violencia" en las escuelas, y las que dan cuenta de manera más pertinente de las violencias cotidianas presentes en las interacciones sociales del sistema escolar (Debarbieux, 1996, 2001, 2002; Carra y Sicot, 1997; Charlot, 1997; Debarbieux, et al., 1999; Viscardi, 2003; Kornblit, 2008; Observatorio Argentino de Violencia en las Escuelas, 2008 y 2010; Kaplan 2009a; García, 2010).

Es en este sentido que se decidió abordar las humillaciones en el ámbito escolar, como un intento por visibilizar lo cotidiano sobre lo excepcional y dar cuenta de uno de los mayores problemas en la escuela. Este texto expone los resultados de una investigación más amplia que analizó las relaciones de humillación entre estudiantes de las escuelas secundarias estatales de la provincia de Buenos Aires (Argentina), para contribuir a la producción de argumentos teóricos, y con base empírica, sobre el problema, desde una perspectiva socioeducativa; particularmente interesa abordar una de las dimensiones trabajadas en la investigación: los atributos a los cuales se refieren las humillaciones; sosteniendo como hipótesis que los términos utilizados durante este tipo de vínculo no son universales, sino que adquieren especificidades y varían en las distintas configuraciones.

Distintos estudios han probado que las humillaciones son una problemática extendida en el ámbito escolar (Merlé, 2006; García, 2010; Suarez Pazos, 2004; D’Angelo y Fernández, 2011) y que constituyen modos de vincularse que inciden negativamente, no solo en las trayectorias y experiencias escolares de los estudiantes, sino en su vida social fuera de la escuela (Merlé, 2006, Kaplan, 2009b). Aunque este tipo de violencia tiene una fuerte presencia en las escuelas, tiende a pasar inadvertida por profesores y directivos (D’Angelo y Fernández, 2011). Así, las humillaciones en la escuela son, desde una perspectiva socioeducativa, un objeto de estudio escasamente abordado ${ }^{2} \mathrm{y}$, a pesar de que en ocasiones los

2 Incluso los trabajos que abordan esta temática se centran principalmente en las relaciones docente-alumnos, siendo insuficientes los estudios que den cuenta de cómo esta modalidad de interacción tiene lugar entre estudiantes. análisis sobre la violencia en la escuela se refieren al tema como una de las modalidades que le afectan, no la abordan como objeto de estudio específico (Debarbieux, 1996 y 2002; Gómez Nashiki, 1996; Charlot, 1997; Kaplan, 2009a; García, 2010).

A pesar de lo dicho, diversos trabajos analizan los motivos que llevan a los estudiantes a discriminar, humillar, hostigar o burlarse de sus pares (García, 2010; Observatorio Argentino de Violencia en las Escuelas, 2014). Esos análisis dan cuenta de las características por las cuales los alumnos son menospreciados por sus compañeros y, en este sentido, resultan un aporte significativo para comprender la problemática; sin embargo, como veremos más adelante, nuestro estudio indaga las características de las humillaciones, teniendo en cuenta los distintos contextos en los cuales se dan estas modalidades de relación, pues entendemos que dichas cualidades son relacionales y, por lo tanto, particulares de cada espacio social.

\section{Las relaciones de humillación en la escuela}

La humillación es una relación social entre una persona (o grupo) que trata a otra como inferior, y esa otra persona (o grupo) se siente agraviada (Kaplan, 2009b). Es un acto y un sentimiento. Humillar tiene su origen en la palabra bumus (tierra), se refiere a bajar, poner en el suelo o rebajar (Diez Mateo, s.f.) y expresa una práctica cultural subyacente de distinción. La humillación supone una diferencia de poder entre grupos interdependientes; un grupo tiene la capacidad de humillar a otro cuando tiene "recursos de poder" más fuertes y, como afirma Elias (2003), solo puede estigmatizar efectivamente si está bien instalado en posiciones de poder a las que el otro grupo tiene negado el acceso: "Es la condición decisiva de toda estigmatización efectiva de un grupo forastero por parte de un grupo establecido" (p. 224). Incluso si el grupo menos forastero dispone de un término estigmatizador para referirse al más poderoso (establecido), éste es inservible porque no causa daño a los miembros (Elias, 2003).

Los términos para estigmatizar otros grupos solo resultan significativos en un contexto de relaciones de poder específicas; su incidencia depende de la conciencia por parte del hablante y del receptor de que la humillación viene respaldada por un grupo poderoso y se dirige a un grupo receptor con recursos de poder más débiles (Elias, 2003). Así, aunque existen importantes cualidades 
desacreditadoras "en casi toda nuestra sociedad" (Goffman, 2006, p. 14), un atributo que estigmatiza a un sujeto puede confirmar la normalidad de otro y no es honroso ni ignominioso en sí mismo. Es posible que haya signos cuyo significado varíe de un grupo a otro, por lo que en realidad se necesita un lenguaje de relaciones, no de atributos.

La tendencia a discutir el problema de la estigmatización social como si se tratase solo de una cuestión de individuos que muestran un profundo rechazo hacia otros, implica percibir únicamente el nivel individual, algo que no puede ser comprendido sin considerar simultáneamente el nivel de grupo (Elias, 2003). La capacidad de un grupo de apuntalar la inferioridad humana del otro grupo, y de hacerla valer, es una función de una figuración específica que ambos grupos forman entre sí; por lo tanto su análisis requiere

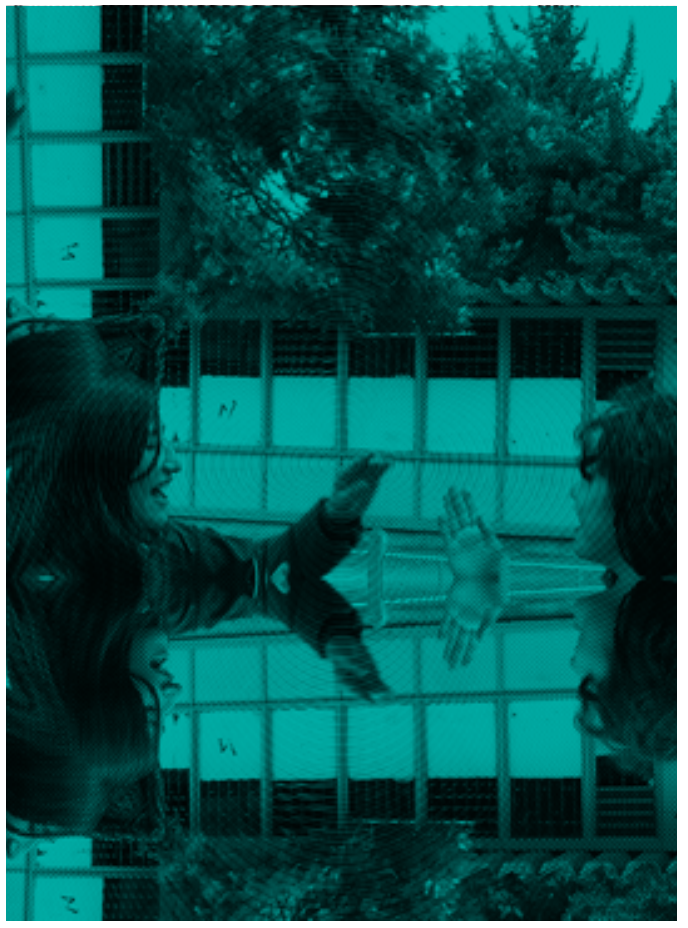

mañana) en dos escuelas estatales urbanas ${ }^{4}$, mixtas, de la provincia de Buenos Aires, que, como veremos, se diferencian entre ellos por los sectores socioeconómicos de pertenencia y las trayectorias educativas. La selección se realizó para analizar las humillaciones en contexto, como un intento de dar cuenta de las diferencias y recurrencias de sus sentidos y prácticas.

Para continuar, es necesario aclarar el término de configuración ${ }^{5}$, retomado de la obra de Elias (1970), quien lo entiende como un estado en el que intervienen grupos interdependientes de personas: los seres humanos están orientados unos con otros y unidos de las más diversas maneras, constituyendo "telas de interdependencia" (p. 15) o configuraciones de muchos tipos, como familias, escuelas, ciudades, estratos o estados ${ }^{6}$; de esa interdependencia se deriva un orden que, al tiempo que es relativamente autónomo frente al orden dominante, es más fuerte y más coactivo que la voluntad y la razón de los individuos aislados que lo constituyen (Elias, 1987). Gracias a su fundamental interdependencia los seres humanos se agrupan siempre en figuraciones específicas: dependen unos de otros y cumplen, unos para otros, unas funciones (Weiler, 1998).

Las acciones de una pluralidad de personas interdependientes se conectan para formar una estructura entretejida con ciertas propiedades, tales como cuotas de poder, ejes de tensión, sistemas de clase y estratificación, deportes, guerras y crisis económicas (Elias y Dunning, 1992). El equilibrio de poder constituye un elemento

naturaleza del problema de investigación llevó a realizar estudio exploratorio a partir de una estrategia de investigación cualitativa (Vasilachis de Gialdino, 2006), por considerar que dicha metodología se ajusta a nuestro interés por contribuir, a partir de datos empíricos, a la elaboración de un saber interpretativo sobre las relaciones de humillación de los estudiantes en el ámbito escolar. Mediante un muestreo intencional se seleccionaron dos grupos de estudiantes de dos cursos de segundo año de secundaria ${ }^{3}$ (turno

3 Los años de secundaria mencionados se refieren a los establecidos con la nueva Ley de Educación Nacional (Ley 26.206) sancionada en 2006. La provincia de Buenos Aires implementó una secundaria de 6 años.
4 Se seleccionaron colegios urbanos, ya que trabajos anteriores como los de Carra y Sicot (1997), Debarbieux, Dupuch y Montoya (1997), Waiselfisz (1998), o Kaplan (2006 y 2009b), advierten que el problema de las violencias en la escuela se encuentra especialmente ligado al ámbito urbano. configuración, figuración y composición se emplean para describir fenómenos semejantes, y este trabajo los utiliza como sinónimos.

6 En este sentido, Elias señala que dicho concepto puede ser aplicado a grupos relativamente pequeños y a sociedades de miles o millones de personas interdependientes.
5 En los escritos consultados en castellano, inglés y portugués los conceptos de 
integral de todas las relaciones humanas; por grandes o pequeñas que sean las diferencias de poder, este equilibrio siempre está presente donde quiera que haya una interdependencia funcional entre personas, el poder:

Es una característica estructural [...] de todas las relaciones humanas $[\ldots]$ Dependemos de otros; otros dependen de nosotros. En la medida en que dependamos de otros más de lo que ellos dependen de nosotros, tienen poder sobre nosotros, independientemente de que hayamos caído bajo su dependencia porque ellos emplearon la fuerza o por nuestra necesidad de ser amados, nuestra necesidad de dinero, consuelo, estatus, una carrera o, simplemente, de emoción (Elias y Dunning, 1992, p. 21).

La investigación tuvo como objetivo general comprender las relaciones de humillación entre estudiantes en la escuela secundaria desde su perspectiva. Interesaba, entre otras cuestiones, caracterizar y comparar las relaciones de humillación en configuraciones particulares que consideraron dimensiones como: prácticas, sentimientos y motivaciones ligadas a la humillación; los atributos o cualidades a los cuales se refieren; las respuestas de los estudiantes que son destinatarios y las intervenciones de escuela ante las humillaciones. Como se ha dicho, se dará cuenta de una de estas dimensiones: los atributos que se utilizan para humillar a los compañeros.

El trabajo de campo se desarrolló en 2011 y partió de una entrevista a las autoridades de las escuelas y de una consulta de su documentación (proyecto institucional), con el objetivo de conocer mejor a la institución y su población; luego se seleccionaron los dos grupos escolares, uno de cada escuela. La recolección de datos se hizo a través de entrevistas en profundidad, individuales y grupales, a los estudiantes de los grupos ${ }^{7}$ con una guía semi-estructurada ${ }^{8}$; además se realizaron observaciones participantes para ahondar en los relatos. Se entrevistó a "tres columnas" (Sirvent, 2003) un total de 51 estudiantes usando esta técnica porque permite "conocer la perspectiva del sujeto estudiado, comprender sus categorías mentales, sus interpretaciones, sus percepciones y sentimientos, los motivos de sus actos" (Corbetta, 2007, p. 344). Se quiso entender estas formas de violencia desde la voz de los estudiantes, teniendo en cuenta que sus percepciones están construidas socialmente y situadas socio-históricamente.

7 Las entrevistas eran optativas.

8 Los nombres de los estudiantes se han modificado para resguardar su anonimato.
Las técnicas de análisis de información empírica se dieron en función de las dimensiones establecidas en los objetivos; a partir de allí se fueron identificando las categorías cercanas a lo empírico y con ellas surgieron otras con mayor grado de abstracción. El programa Atlas.ti., fue empleado para estudiar los datos recogidos y la indagación se realizó en un ida y vuelta entre la teoría y lo empírico, buscando poner en tensión nuestras premisas teóricas y los datos del trabajo de campo.

\section{Configuraciones analizadas}

La primera configuración analizada fue un grupo escolar que llamamos 1, conformado por alumnos de un curso de segundo año de secundaria de una escuela estatal céntrica que, creada durante la década del 60, tiene como objetivo la inclusión de estudiantes con trayectorias educativas interrumpidas (abandono, repitencia) y con dificultades para respetar los marcos disciplinarios escolares. El grupo de 22 estudiantes, 13 varones y 9 mujeres, fue entrevistado en su totalidad; la mayoría de ellos pertenece a sectores socioeconómicos bajos y medios bajos y la repitencia atraviesa sus trayectorias educativas: 14 habían repetido al menos un año escolar ( 8 una vez, 4 dos veces, 1 tres veces y 1 cuatro veces) y el grupo era entendido por docentes, directivos, preceptores y los propios alumnos, como "difícil", con un bajo rendimiento académico y problemas de comportamiento y trato con las autoridades.

La segunda configuración (2) estuvo integrada por estudiantes de un curso de segundo año (turno mañana) de educación secundaria, pertenecientes a una escuela estatal céntrica y tradicional con casi 100 años de historia, una de las entidades más solicitadas y prestigiosas de la zona. Se entrevistaron 19 de los 36 alumnos del grupo, en su mayoría de clase media, 18 mujeres (se entrevistaron 15) y 18 varones (se entrevistaron 14); solo uno de los 29 educandos había repetido un año, y esta baja tasa de repitencia se asocia en cierta medida a la política institucional de que quienes repiten pierden su cupo en el turno de la mañana, pasando al turno de la tarde o siendo expulsados de la escuela. En palabras de un directivo: "En esta escuela... repetidores nunca quisieron". En cuanto al vínculo entre estudiantes y autoridades de la institución, los alumnos afirman que por lo general se comportan con respeto cuando se dirigen a ellas, en sus relatos incluso se advierte una especie de sumisión. 


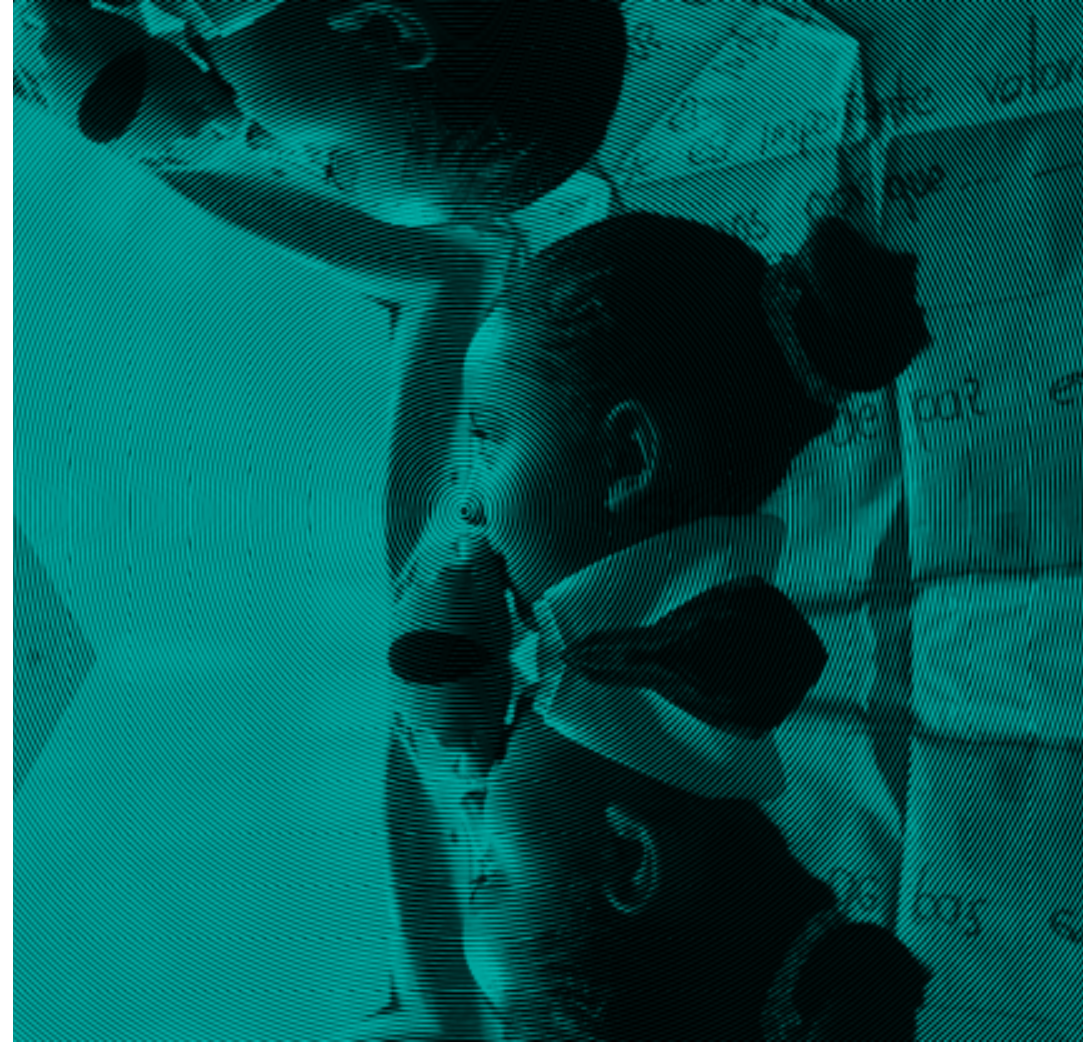

llar, el análisis destaca diferencias y contrastes entre los dos grupos escolares, que son esenciales para entender el hecho en contexto. En primer lugar, se destacan las humillaciones vinculadas al nivel socioeconómico. Los entrevistados del grupo escolar 1 (sectores bajos y medios bajos) señalaron que frecuentemente las características que llevan a humillar a los compañeros se relacionan con pertenecer a sectores socioeconómicamente más acomodados: Tener plata, ropa cara o vivir en barrios "chetos" son características que dan lugar a la inferiorización de los alumnos por parte de sus pares.

Esto contrasta con lo manifestado por los estudiantes del grupo 2 (sectores medios), quienes afirman que a menudo se menosprecia a los compañeros desde alusiones a la pertenencia a sectores socioeconómicos bajos, destacando cualidades como "ser pobre", "no poder comprar zapatillas o ropa de marca" o "vivir en la villa". En ambas configuraciones, las modalidades de sociabilidad se refieren al nivel socioeconómico de los estudiantes, sin embargo, los sentidos en uno y en otro son muy distantes.

Algo similar ocurre con el rendimiento escolar y las calificaciones. En el grupo escolar 1 el acto de estudiar, saber el contenido escolar y sacar buenas calificaciones es válido como atributo plausible de humillación ("las joden con ser nerds"; "te comés los libros"; "la rebajan porque sabe todo"; "saben que estudio y peor, van a molestarme por eso"); mientras que, por el contrario,

9 Se refiere a las villas de emergencia, donde viven los sectores socioeconómicos más humildes.

en el grupo 2 las bajas notas y no saber el contenido escolar son los motivos para estigmatizar al otro.

Claudia: A veces los chicos... los cargan, le dicen "¡Ah!, sos un burro", "¡Ay! la prueba fue tan fácil y vos te sacaste mal”, y es horrible (...) Te empiezan a cargar por la nota (Configuración 2).

Guillermina comenta que en una clase de Geografía una docente le preguntó por un continente y ella contestó "España". Desde ese momento los compañeros la burlan llamándola "España" (Configuración 2).

Las diferencias en los términos utilizados para menospreciar a los compañeros se observaron también en los vínculos de los alumnos con las autoridades de la escuela; en el grupo escolar 1 los estudiantes son humillados por sus pares por mantener una buena relación con docentes o directivos, incluso por seguir sus indicaciones; el segundo grupo no presenta esa situación.

Karina: Cuando se están peleando dos chicos te dicen "Andá a llamar a la preceptora". Y vos quedás como la re buchona ${ }^{10}(\ldots) \mathrm{Y}$ después te bardean todos, te dicen cualquier cosa: "Andá vos, mula ${ }^{11}$, buchona, chupamedia ${ }^{12}$ ", de todo (Configuración 1).

Emilio: Quedás un poco humillado porque le estás haciendo caso a un profesor y muchos dicen: "Ay, le tuvo que hacer caso a este profesor de mierda” (Configuración 1).

\section{Chismoso.}

11 Persona que es mandada por otro.

12 Obsecuente. 
Esta información permite concluir que los atributos implícitos en las humillaciones no son universales, sino que cada configuración presenta cualidades particulares por las cuales un sujeto humilla a otro. Desde nuestra perspectiva, y siguiendo a autores como Norbert Elias y Pierre Bourdieu ${ }^{13}$, consideramos que las percepciones y prácticas de los individuos están atravesadas por las condiciones objetivas en las que se sitúan. Los sujetos están marcados por circunstancias objetivas que funcionan a la vez como condiciones limitantes y como puntos de referencia para sus emociones y actuaciones. Las humillaciones también están atravesadas por las características de la configuración, por las interdependencias propias de cada grupo escolar, que dan lugar a valoraciones específicas. Así, aunque algunos atributos por los cuales los estudiantes son humillados coinciden en ambos grupos, no se puede afirmar que las cualidades sobre las que se asientan son similares en los distintos contextos.

La segunda cuestión a subrayar, estrechamente vinculada con lo anterior, es que los atributos que tienen presencia en uno u otro grupo se definen en función de las relaciones de fuerza. La sociodinámica de la relación (Elias, 2003), entre quienes humillan y los humillados, viene determinada por la forma de su vínculo, no por las características de los grupos afectados. Las cualidades por las cuales unos estudiantes o grupos de estudiantes humillan a otros, se definen en función de quiénes ocupan las posiciones dominantes y quiénes no en ese grupo escolar particular, o, en palabras de Elias (2003), en función de quiénes se encuentran bien instalados en posiciones de poder a las que el otro no tiene acceso.

En el grupo escolar 1 (clase baja y media baja) los entrevistados afirman que quienes ostentan posiciones dominantes se identifican, por un lado, con pertenecer a sectores sociales de bajos ingresos (vivir en villas y barrios populares, usar vestimenta asociada con lo humilde -gorrita, aros y ropa holgada-) y por el otro, con tener mala conducta y mal desempeño escolar. Los más respetados, o con posición de liderazgo, son asociados con tipologías que podríamos denominar "marginales", en el sentido de que se alejan de ciertas normas valoradas como positivas en la escuela, como tener buenas notas, respetar a docentes y directivos, tener buena posición económica, etc. Es el grupo de "los chicos de la ventana" o de los "quilomberos", que faltan el respeto a los profesores, golpean a sus pares, gritan, se pegan entre ellos y consumen drogas. Ellos

13 Las teorías de estos autores no se desarrollan en este escrito por una cuestión de espacio. asumen esta caracterización definiéndose como los "peores de la clase"; el líder es Leandro y es temido por algunos estudiantes.

E: ¿Por qué creés que Leandro es el líder?

Tatiana: Leandro pega dos gritos en el salón y se callan todos [...] la mayoría como lo ven así... él todo el tiempo está hablando de cosas, de que va a robar y todo eso, capaz le tienen miedo [...] Se piensa que el vivir en un barrio de negros, que vistiéndose así, que robar y todo eso, es ser mejor que los demás.

Son los estudiantes que se identifican con estos rasgos los que humillan a sus pares y los que no son humillados.

Los entrevistados también mencionaron que quienes ocupan posiciones menos legitimadas presentan o se identifican con cualidades opuestas a las de los más respetados, como "tener buena conducta"; "tener más plata"; "saber todo (el contenido escolar)"; "sacarse buenas notas". Estos alumnos son señalados por sus compañeros como los menos respetados o valorados del curso.

$\mathrm{E}:$ ¿Y quién sería el menos respetado del curso?

Marcos: Y... los que se portan bien, los que nunca molestan, nada. Damián: Acá a los que no estudian los valoran más.

La identificación con esas "características marginales" se debe en parte a que los jóvenes las vinculan con la posibilidad de generar miedo en el resto de compañeros. Puede pensarse que existe una estética y unas cualidades marginales asociadas a la taxonomía de joven violento, y que para ciertos estudiantes resultan prestigiosas. En un estudio anterior (Kaplan, Krotsch y Orce, 2012) se observan hallazgos que refuerzan la idea del estrecho vínculo entre atributos como vestirse con ropa deportiva o vivir en barrios humildes, y ser considerado violento en el discurso de los jóvenes.

Vivencias, estéticas y conductas que no son valores positivos hegemónicos de la sociedad, sino estimaciones prestigiosas para ciertos estudiantes, acentuadas por ellos en su práctica cotidiana: vestirse con gorrita, arito y ropa holgada emulando cierto estereotipo humilde; la realización de actividades delictivas; el consumo de drogas o vivir en barrios populares. Lo que se observó es que características que en nuestra sociedad se pueden considerar como "marginales" son asumidas como prácticas jerarquizantes por ciertos alumnos.

En consonancia con lo que plantea Bourgois (2010), muchos jóvenes eligen construir la marca de identidad sobre atributos que la sociedad desvaloriza: robo, delitos, consumo de drogas; lo que les permite subvertir el orden social aunque solo sea en su red de relaciones. Las relaciones de humillación establecidas por los estudiantes con sus compañeros podrían estar siendo utilizadas por algunos jóvenes como mecanismo de desafío y resistencia a los 
valores hegemónicos, tanto escolares (buen desempeño académico, respeto por las normas y autoridades), como sociales (pertenencia a sectores sociales y económicos acomodados).

En el grupo escolar 2 quienes ostentan las posiciones dominantes se identifican con características como tener una buena posición socioeconómica, altas calificaciones o saber el contenido escolar. El grupo más respetado, aquel que los entrevistados identifican como el "más popular", está constituido por alumnos de clase media con un buen desempeño escolar (la alumna más estudiosa y con mejores calificaciones del curso pertenece a este grupo).

Micaela: Es popular por tener cosas caras, de moda.

Claudia: Porque se viste bien se juntan con esa para ser más populares y porque una sea más humilde no se juntan.

Las características de quienes ostentan las posiciones dominantes y quienes no, de los que humillan y son humillados, varían en los dos grupos escolares analizados. En parte ello podría explicar las diferencias en cada configuración sobre las cualidades que sustentan las humillaciones.

\section{Conclusiones}

El análisis realizado es un importante punto de partida en el intento de dar cuenta de la manera en que los atributos que susten$\tan$ las humillaciones, antes que ser cualidades universales similares en los distintos contextos, están definidos por las particularidades de las configuraciones donde estas relaciones tienen lugar, por los recursos de poder de los grupos en cada configuración; por la diferencia de poder entre ellos (Elias, 2003). Las características de los humillados y de los que humillan se definen por la posición que ocupan en el marco de unas relaciones de poder determinadas.

Lo que nos interesa subrayar es que las características de las humillaciones, y de los lugares que los sujetos ocupan en esa relación social, están determinadas por los vínculos y relaciones de fuerza que se establecen entre los grupos, al interior de cada configuración, y no por las cualidades de los individuos con independencia de ellos. Las humillaciones deben ser comprendidas en su dimensión relacional; por lo tanto, se pierde de vista la clave del problema cuando se indaga exclusivamente en la estructura de personalidad de sujetos concretos.

Este enfoque disputa sentidos a aquellos estudios que abordan la temática de la violencia en la escuela desde una mirada individualizante y se concentran en el acosador y en la víctima (también en el grupo de alumnos o en sus familias), describiendo las características particulares (sobre todo los atributos psicológicos) que, por lo general, tienen quienes asumen estos roles, sin considerar el contexto que da lugar a estas interacciones. Así mismo, acarrea el peligro de focalizar las soluciones en los estudiantes involucrados, patologizándolos, y de desresponsabilizar a la escuela y a las mediaciones que realiza. 


\section{Referencias}

Bourgois, P. (2010). En busca de respeto: vendiendo crack en Harlem. Buenos Aires: Siglo XXI.

Brener, G., y Kaplan, C. (2006). Violencias, escuela y medios de comunicación. En Kaplan, C. (Dir.), Violencias en plural: Sociologia de las violencias en la escuela. Buenos Aires: Miño y Dávila, pp. 77-101.

Carra, C., y Sicot, F. (1997). Una autre perspective sur les violences scolaires: L'expérience de victimation. En Charlot, B., y Emin, J. (Coords.), Violences a l'ecole - etat des savoirs. París: Armand Colin Mason, pp. 61-82.

Charlot, B. (1997). Introduction. Violences scolaires: Représentations, pratiques et normes. En Charlot, B., y Emin, J. (Coords.), Violences a l'école: État des savoirs. París: Armand Colin, pp. 1-13.

Corbetta, P. (2007). Metodología y técnicas de la investigación social. Madrid: MacGrawHill.

D’Angelo, L., y Fernández, D. (2011). Clima, conflictos y violencia en la escuela. Buenos Aires: Fondo de las Naciones Unidas para la Infancia (UNICEF), Facultad Latinoamericana de Ciencias Sociales (FLACSO).

Debarbieux, E. (1996). La violence en milieu scolaire: I- État des lieux. París: ESF éditeur.

Debarbieux, E. (2001). A violência na escola francesa: 30 anos de construção social do objeto (1967-1997). Educação e Pesquisa (Brasil), Vol. XXVII, No. 1, pp. 163-193.

Debarbieux, E. (2002). Cientistas, políticos e violência: rumo a uma comunidade científica européia para lidar com a violência nas escolas? En Debarbieux, E., y Blaya, C. (Coords.), Violência nas escolas: Dez abordagens européias. Brasília: Unesco, pp. 13-33.

Debarbieux, E., Dupuch, A., y Montoya, Y. (1997). Pour en finir avec le handicap socio-violent: une aproche comparative de la violence en milieu scolaire. En Charlot, B., y Emin, J. (Coords.), Violences à $\square$ école: État des savoirs. Paris: Armand Colin, pp. 17-40.

Debarbieux, E., Garnier, A., Montoya, Y., y Tichit, L. (1999). La violence en milieu scolaire: Le désordre des choses, Vol. 2. París: ESF éditeur.
Díez Mateo, F. (s.f.). Diccionario español etimológico del siglo XX. (S.C.): Academo.

Elias, N. (1970). Introdução à Sociologia. Lisboa: Edições 70.

Elias, N. (1987). El proceso de la civilización: Investigaciones sociogenéticas y psicogenéticas. Madrid: Fondo de Cultura Económica.

Elias, N. (2003). Ensayo acerca de las relaciones entre establecidos y forasteros. Reis. Revista española de investigaciones sociológicas (España), pp. 219-251.

Elias, N., y Dunning, E. (1992). Deporte y ocio en el proceso de la civilización. México: Fondo de Cultura Económica.

García, S. (2010). Violencias, incivilidades y miedos en el ámbito educativo: Un estudio socioeducativo sobre la perspectiva de los estudiantes de educación secundaria, Tesis de Maestría, Escuela de Educación, Universidad de San Andrés, Buenos Aires (Argentina).

Goffman, E. (2006). Estigma: La identidad deteriorada. Buenos Aires: Amorrortu.

Gómez Nashiki, A. (1996). La violencia en la escuela primaria. Tesis de Maestría en Sociología Política, Instituto de Investigaciones Dr. José María Luis Mora. (México).

Kaplan, C. (2006). Violencias en plural: Sociología de las violencias en la escuela. Buenos Aires: Miño y Dávila.

Kaplan, C. (2009a). Violencia escolar bajo sospecha. Buenos Aires: Miño y Dávila.

Kaplan, C. (2009b). La humillación como emoción en la experiencia escolar. En Kaplan, C., y Orce, V. (Coords.), Poder, prácticas sociales y proceso civilizador: Los usos de Norbert Elias. Buenos Aires: Novedades Educativas, pp.99-108.

Kaplan, C., Krotsch, L., y Orce, V. (2012). Con ojos de joven: Relaciones entre desigualdad, violencia y condición estudiantil. Buenos Aires: Editorial de la Facultad de Filosofía y Letras, Universidad de Buenos Aires. 
Kornblit, A. L. (2008). Violencia escolary climas sociales. Buenos Aires: Biblos.

Merlé, P. (2006). L'humiliation des élèves: une pratique anti-pédagogique. Éducation \& Management, (31), p. 32. Obtenido desde http://www.educrevues.fr $/ \mathrm{EM} /$ AffichageDoc ument.aspx?iddoc $=34382$

Observatorio Argentino de Violencia en las Escuelas. (2008). Violencia en las escuelas: Un relevamiento desde la mirada de los alumnos. Buenos Aires: Ministerio de Educación de la Nación.

Observatorio Argentino de Violencia en las Escuelas. (2010). La Violencia en las escuelas: Un relevamiento desde la mirada de los alumnos II. Buenos Aires: Ministerio de Educación de la Nación.

Observatorio Argentino de Violencia en las Escuelas. (2014). Relevamiento estadístico sobre clima escolar, violencia y conflicto en escuelas secundarias según la perspectiva de los alumnos. Buenos Aires: Ministerio de Educación de la Nación.
Sirvent, M. T. (2003). El Proceso de Investigación. Investigación y Estadística I. Buenos Aires: Oficina de Publicaciones de la Facultad de Filosofía y Letras (Opfyl).

Suárez Pazos, M. (2004). Los castigos y otras estrategias disciplinarias vistos a través de los recuerdos escolares. Revista de Educación, No. 335, pp. 429-443.

Vasilachis de Gialdino, I. (2006). La investigación cualitativa. Estrategias de investigación cualitativa. Barcelona: Gedisa, pp. 23-64.

Viscardi, N. (2003). Enfrentando la violencia en las escuelas: Un informe de Uruguay. En Werthein, J., Cecilia Braslavsky, C., Tedesco, J., Ouane, A., y Da Cunha, C. (Coords.), Violencia na escola: América Latina e Caribe. Brasília: UNESCO, pp. 153-205.

Waiselfisz, J. J. (1998). Juventude, violencia e cidadania: Os jovens de Brasilia. San Pablo: UNESCO, Cortez Editora.

Weiler, V. (1998). Figuraciones en proceso. Bogotá: Fundación Social. 